\title{
Microscope imaging mass spectrometry with a reflectron
}

Cite as: Rev. Sci. Instrum. 91, 023306 (2020); https://doi.org/10.1063/1.5142271

Submitted: 11 December 2019 . Accepted: 13 January 2020 . Published Online: 04 February 2020

Robert J. Burleigh, Ang Guo, Natasha Smith, Andrew Green, Steve Thompson, (D) Michael Burt, and (iD) Mark Brouard

\section{ARTICLES YOU MAY BE INTERESTED IN}

Developing a camera-based 3D momentum imaging system capable of $1 \mathrm{Mhits} / \mathrm{s}$

Review of Scientific Instruments 91, 023316 (2020); https://doi.org/10.1063/1.5138731

Multiple-ion-ejection multi-reflection time-of-flight mass spectrometry for singlereference mass measurements with lapping ion species

Review of Scientific Instruments 91, 023201 (2020); https://doi.org/10.1063/1.5131582

Velocity map imaging of ions and electrons using electrostatic lenses: Application in photoelectron and photofragment ion imaging of molecular oxygen

Review of Scientific Instruments 68, 3477 (1997); https://doi.org/10.1063/1.1148310

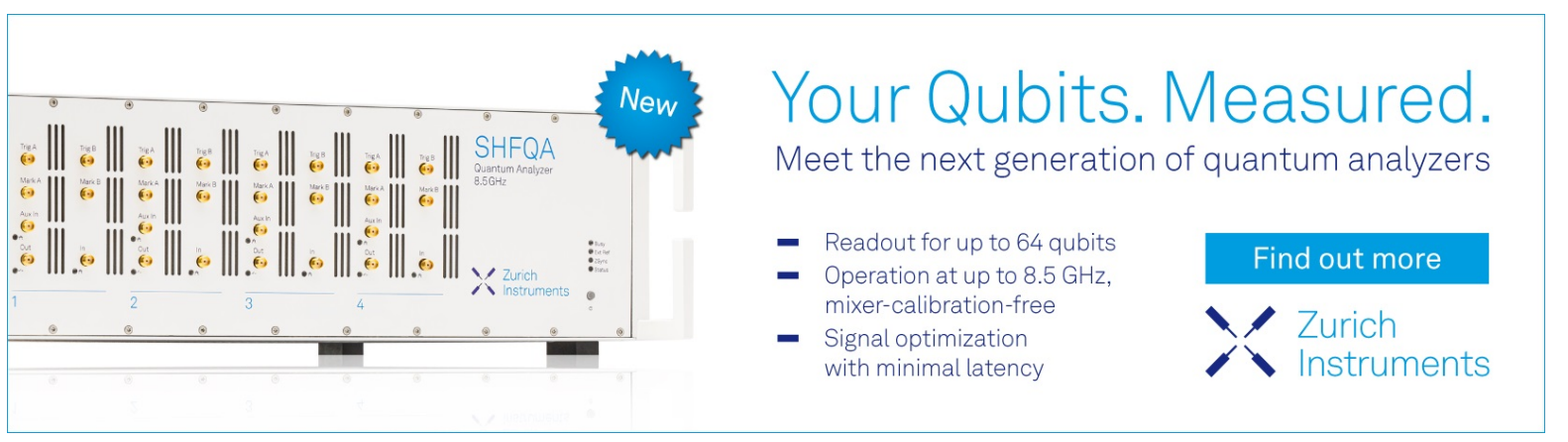




\title{
Microscope imaging mass spectrometry with a reflectron
}

\author{
Cite as: Rev. Sci. Instrum. 91, 023306 (2020); doi: 10.1063/1.5142271 \\ Submitted: 11 December 2019 • Accepted: 13 January 2020 • \\ Published Online: 4 February 2020
}

\author{
Robert J. Burleigh,' Ang Guo,' Natasha Smith,' Andrew Green,' Steve Thompson, \\ Michael Burt, ${ }^{7, a)}$ (iD) and Mark Brouard ${ }^{1, b)}$ (iD)
}

\begin{abstract}
AFFILIATIONS
${ }^{1}$ The Chemistry Research Laboratory, Department of Chemistry, University of Oxford, 12 Mansfield Road, Oxford OX1 3TA, United Kingdom

${ }^{2}$ Ionoptika Limited, Unit B6, Millbrook Close, Chandler's Ford, Eastleigh SO53 4BZ, United Kingdom
\end{abstract}

a) Electronic mail: michael.burt@chem.ox.ac.uk

b) Author to whom correspondence should be addressed: mark.brouard@chem.ox.ac.uk

\begin{abstract}
A time-of-flight microscope imaging mass spectrometer incorporating a reflectron was used to image mass-resolved ions generated from a $270 \mu \mathrm{m}$ diameter surface. Mass and spatial resolutions of $8100 \pm 700 \mathrm{~m} / \Delta \mathrm{m}$ and $18 \mu \mathrm{m} \pm 6 \mu \mathrm{m}$, respectively, were obtained simultaneously by using pulsed extraction differential acceleration ion optical focusing to create a pseudo-source plane for a single-stage gridless reflectron. The obtainable mass resolution was limited only by the response time of the position-sensitive detector and, according to simulations, could potentially reach $30200 \pm 2900 \mathrm{~m} / \Delta \mathrm{m}$. The spatial resolution can be further improved at the expense of the mass resolution to at least $6 \mu \mathrm{m}$ by increasing the applied extraction field. An event-triggered fast imaging sensor was additionally used to record ion images for each time-of-flight peak resolved during an experimental cycle, demonstrating the high-throughput capability of the instrument.
\end{abstract}

Published under license by AIP Publishing. https://doi.org/10.1063/1.5142271

\section{INTRODUCTION}

Ion microscope mass spectrometry imaging (MSI) enables high-throughput recording of mass-resolved chemical distributions. This method uses defocused laser or primary ion pulses to simultaneously ionize a sample, creating an object plane of ions that is separated by time-of-flight and electrostatically magnified onto the image plane of a position-sensitive detector. ${ }^{1-5}$ Microscope MSI, therefore, allows mass-resolved ion images to be sequentially recorded and, when paired with multi-mass imaging sensors, offers an efficient alternative to conventional microprobe imaging techniques, which instead create images pixel by pixel. ${ }^{6-14}$ Despite this advantage, electrostatic focusing precludes ion trapping, lowering its attainable mass resolution relative to microprobe MSI. To address this, we present and characterize a microscope imaging mass spectrometer that incorporates a reflectron capable of obtaining ion images with simultaneously high mass and spatial resolutions.

Focusing ion images through non-linear trajectories can be accomplished with minimal time-of-flight distortion. Schueler demonstrated a secondary ion microscope that employed three $90^{\circ}$ electrostatic analyzers to resolve images to $8000 \mathrm{~m} / \Delta \mathrm{m}$ and $1 \mu \mathrm{m}$ over an essentially limitless mass range, and without first-order chromatic aberrations. ${ }^{4,15}$ Follow-on designs employing matrixassisted laser desorption/ionization have provided comparable results. ${ }^{5,7,10-12}$ Hazama and co-workers later characterized a multiturn ion microscope that used four electrostatic analyzers to cycle ions a set number of turns before detection, significantly separating their times-of-flight. ${ }^{16-18}$ With this instrument, a mass resolution of $130000 \mathrm{~m} / \Delta m$ over a window of $8 \mathrm{Da}$ was reported after 500 cycles for the angiotensin II ion. However, images recorded in this way required sensitive alignment of the ion optics, and aberrations were noted after one cycle, contrary to simulations that demonstrate this optical arrangement provides third-order focusing.

Reflectrons combine the advantages of the above techniques by offering the potential to record mass- and spatially resolved ion images over wide mass ranges. They routinely achieve mass resolutions in the $10^{5}-10^{6} \mathrm{~m} / \Delta m$ range depending on whether they are in single- or multi-reflecting configurations and correct for the 
time-of-flight dependence observed when ions with the same massto-charge ratio $(\mathrm{m} / \mathrm{z})$ are created with different kinetic energies. ${ }^{19-24}$ For example, single- and dual-stage reflectrons provide first- and second-order energy focusing, respectively. Reflectrons also show particular promise for ion imaging. Leskiw and co-workers recorded velocity-mapped ion images through a reflectron, indicating that the initial spatial distribution of ions can be similarly preserved using different ion optical potentials. ${ }^{25}$ Yavor and co-workers additionally noted that multi-reflecting time-of-flight instruments can satisfy the stigmatic imaging condition. ${ }^{24}$ Collectively, these developments open the possibility of performing microscope imaging at mass resolutions comparable to those achieved by ion traps.

This report experimentally establishes a proof of concept for stigmatic imaging with a reflectron by coupling one with a time-of-flight microscope imaging mass spectrometer. The instrument can be considered as a two-stage time-of-flight arrangement where the image plane of the linear ion microscope doubles as a pseudo-source for the reflectron. This plane is created using the post-extraction differential acceleration (PEDA) technique, which immediately extracts surface ions following ionization to retain their spatial information and which corrects for their initial velocity distributions by raising the potential of the extraction electrode after they pass by. ${ }^{26,27}$ In this way, ions of a particular $m / z$ with slower initial velocities gain more kinetic energy from the raised potential than faster ions, creating a temporal focal plane with high mass resolution beyond the ion microscope. This plane is then deflected through a single-stage reflectron sector that is configured to satisfy its energyfocusing condition ${ }^{23}$ and projected onto a position-sensitive detector. As will be demonstrated below, this focusing arrangement provides simultaneously high mass and spatial resolutions over a $\sim 300$ Da window.

\section{EXPERIMENTAL SECTION}

A single-stage gridless reflectron was joined to a home-built time-of-flight microscope imaging mass spectrometer to increase its resolution beyond $3000 \mathrm{~m} / \Delta \mathrm{m}^{27,28}$ Figure 1(a) illustrates how the resulting instrument focuses ions generated from a sample surface

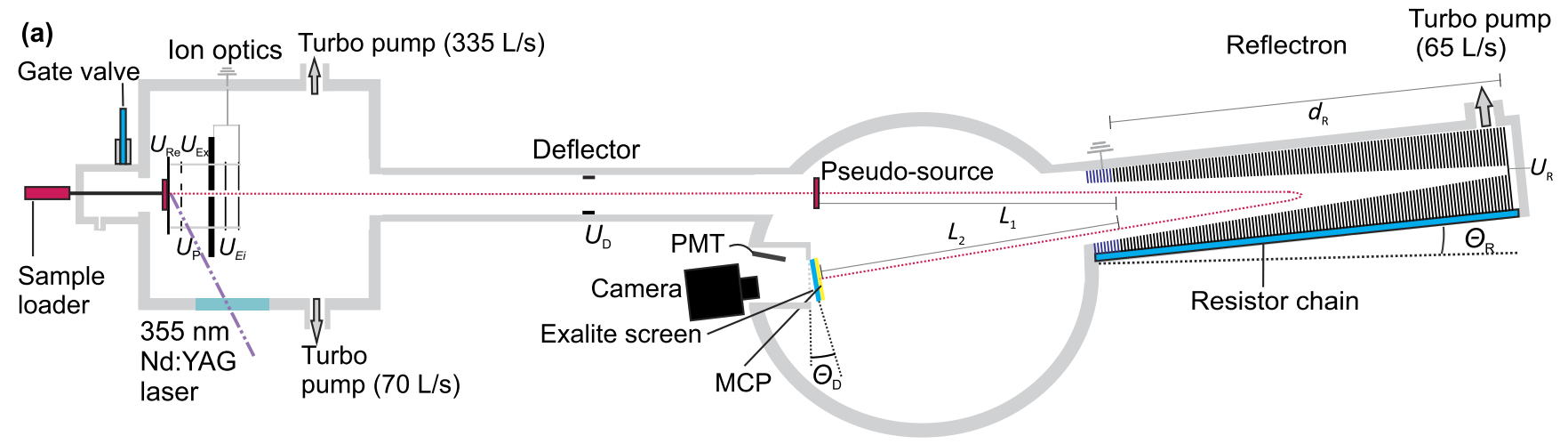

(b)

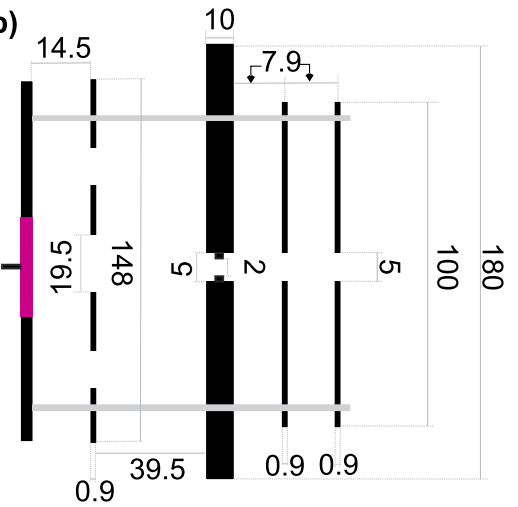

(c)

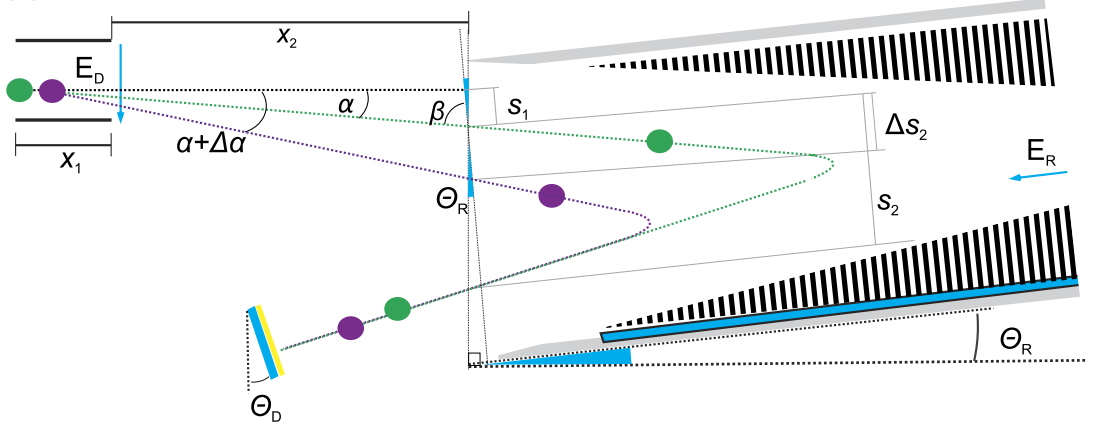

FIG. 1. (a) A schematic of the microscope imaging mass spectrometer. The red trace represents a typical ion trajectory that originates following laser-induced ionization of a sample. The ions are focused by an ion microscope, deflected through a single-stage gridless reflectron, and projected onto a position-sensitive detector. lon images and mass spectra are recorded using an event-triggered imaging sensor or using a photomultiplier tube (PMT) and CCD camera. The adjustable instrument parameters include the ion microscope potentials $\left(U_{R e}, U_{E x}\right.$, and $\left.U_{E \mathrm{Ei}}\right)$, the applied PEDA potential $\left(U_{\mathrm{P}}\right)$, the deflector and reflectron potentials $\left(U_{D}\right.$ and $\left.U_{R}\right)$, and the reflectron and detector angles $\left[\theta_{\mathrm{R}}\right.$ (blue shaded angles) and $\left.\theta_{\mathrm{D}}\right]$. Additional details are provided in the text. (b) The ion microscope electrode dimensions, in millimeters. The highlighted area represents the sample. (c) A diagram illustrating stigmatic imaging of ions deflected through a reflectron. The green and purple traces represent isobaric ions with high and low initial kinetic energies, respectively. For simplicity, the PEDA pseudo-source plane is omitted. To achieve a spatial focus, these ions must travel the same distance perpendicular to the reflectron axis. As detailed in the text, this can be controlled using the angle between the deflected ion beam and the reflectron $\left(\alpha+\theta_{R}\right){ }^{33}$ When focused correctly, each ion will travel a perpendicular distance $s_{1}+s_{2}+\Delta s_{2}$. 
through an ion microscope and drift space (red trace). The ions are deflected into the reflectron and turned toward a detector at the end of a second field-free region. Ions impacting the detector are amplified using dual-stack microchannel plates and converted to photons by an Exalite 404 screen. The response time of this arrangement is $3.7 \mathrm{~ns}{ }^{29}$ Flashes of light corresponding to individual ion events are then recorded simultaneously using an intensified CCD camera and a photomultiplier tube (PMT) or with an event-triggered fast imaging sensor. ${ }^{30-32}$

The ion optical assembly comprises five cylindrical electrodes: a repeller, an extractor, and an einzel lens. Their dimensions are provided in Fig. 1(b). The repeller has four recesses, in which $25 \mathrm{~mm}$ $\times 25 \mathrm{~mm} \times 1.2 \mathrm{~mm}$ conductive plates can be placed, and can be rotated to align the center of each plate perpendicular to the timeof-flight axis. In the experiments reported here, ions were laser desorbed from indium tin oxide surfaces coated with commercial dyes using a $10 \mathrm{~Hz} 355 \mathrm{~nm} \mathrm{Nd:YAG} \mathrm{laser} \mathrm{(Continuum} \mathrm{Powerlight} \mathrm{8010)}$ defocused to cover approximately $10 \mathrm{~mm} \times 10 \mathrm{~mm}$ of the sample. The ions were then immediately extracted to retain their initial spatial information and focused into an acceleration region bounded by the extractor and einzel lens entrance.

The extractor potential is set using the single-field PEDA technique; a baseline potential extracts ions from the surface following ionization and is raised after a set delay using a high voltage switch (Behlke HTS 121) once a desired $\mathrm{m} / \mathrm{z}$ packet has entered the acceleration region. ${ }^{26}$ For a particular $\mathrm{m} / z$, slower ions penetrate a shorter distance beyond the extractor at the PEDA pulse time than faster ions and experience a greater increase in kinetic energy from the potential rise on the extractor. This difference corrects for the initial velocity distribution of the ions and creates a temporal focus plane that is used as the pseudo-source for the reflectron. The PEDA pulse is timed to focus the chosen $\mathrm{m} / \mathrm{z}$, and the effective mass range of the instrument is centered around this focal point. Many-field or electrodynamic PEDA variants can extend this range but were not needed to characterize the mass resolution of the instrument presented here. $^{27}$

After exiting the einzel lens, the ions travel $721 \mathrm{~mm}$ before being deflected into the reflectron by a pair of $30 \mathrm{~mm}$ long parallel plate electrodes. The axial distance between the end of the ion optics assembly and the entrance of the reflectron is $1436 \mathrm{~mm}$. The reflectron itself comprises 65 cylindrical electrodes separated by $10 \mathrm{~mm}$ ceramic spacers for a total length of $569 \mathrm{~mm}$. The first eight plates in the reflectron assembly are grounded, and the remaining electrodes are connected to a printed circuit board containing a resistor chain that creates a linearly decreasing potential gradient. The electrodes in the reflectron have linearly decreasing irides, starting at $90 \mathrm{~mm}$ and finishing as a solid plate where the reflectron potential is applied.

The angle between the reflectron axis and deflected ion beam is crucial for stigmatic imaging. ${ }^{33}$ Figure 1 (c) illustrates that isobaric ions with distinct kinetic energies enter the reflectron at angles that depend on their flight times through the deflector. For a constant deflection field $E_{\mathrm{D}}$ with axial length $x_{1}$, the deflection angle $\alpha$ is shallower for faster ions, meaning that, relative to the reflectron axis, they enter the ion mirror at a smaller distance from the initial (undeflected) time-of-flight axis than slower ions. As these faster ions also penetrate deeper into the reflectron, they sweep a larger distance perpendicular to its axis during the time $t_{\mathrm{R}}$ they remain in its field
$E_{\mathrm{R}}$, and vice versa. To achieve a stigmatic image, the total length $s$ perpendicular to the reflectron axis must be constant with respect to the initial kinetic energy $U$ of each ion in the packet. This can be carried out to the first order when $\mathrm{d} s / \mathrm{d} U=0$, where $s$ can be defined geometrically using Fig. 1(c) as $s_{1}+s_{2}+\Delta s_{2}$ or $^{33}$

$$
s=\frac{x_{2} \tan \alpha}{\cos \theta_{\mathrm{R}}}+v t_{\mathrm{R}} \sin \left(\alpha+\theta_{\mathrm{R}}\right) .
$$

In Eq. (1), $x_{2}, \theta_{\mathrm{R}}$, and $v$ represent the axial drift distance between the deflector and reflectron entrance, the angle between these axes, and the instantaneous velocity of an ion entering the reflectron, respectively. The flight time spent in a single-stage reflectron has previously been established as ${ }^{2}$

$$
t_{\mathrm{R}}=\frac{2}{E_{\mathrm{R}}} \sqrt{\frac{2 m U}{z}} .
$$

By defining $\beta=\frac{\pi}{2}-\alpha-\theta_{\mathrm{R}}$ and noting that $\alpha\left(U, E_{\mathrm{D}}, x_{1}\right)$, Eq. (2) can be used to express $s$ as $s\left(U, E_{\mathrm{D}}, E_{\mathrm{R}}, x_{1}, x_{2}, \theta_{\mathrm{R}}\right)$,

$$
s=\frac{x_{1} x_{2}}{2 U \cos \theta_{\mathrm{R}}} E_{\mathrm{D}}+\left(\frac{4 U^{2}+x_{1}^{2} E_{\mathrm{D}}^{2}}{U E_{\mathrm{R}}}\right) \sin \beta \cos \beta .
$$

Stigmatic images can, therefore, be focused by carefully adjusting $s$ using the mass spectrometer configuration $\left(x_{1}, x_{2}, \theta_{\mathrm{R}}\right)$ or the relevant electrode potentials $\left(U, E_{\mathrm{D}}, E_{\mathrm{R}}\right)$.

Ions exiting the reflectron travel through a $673 \mathrm{~mm}$ field free region and impact the $25 \mathrm{~mm}$ diameter microchannel plate/Exalite 404 assembly described above. The externally controllable detector angle $\theta_{\mathrm{D}}$ is set to be perpendicular to the impinging ion trajectories. The resulting flashes of light are recorded either with a photomultiplier tube and an intensified CCD camera (Photonic Science) or with an event-triggered Pixel Imaging Mass Spectrometry (PImMS) camera installed with the PImMS1 sensor. ${ }^{30}$ The latter is a time-stamping device that allows the position of a detected event to be assigned with a time-of-flight. The PImMS camera can record continuously for $50 \mu$ s with a timing precision of $12.5 \mathrm{~ns}$, allowing multi-mass imaging during a single experiment. This provides an advantage over the CCD camera, which can only produce an integrated image of a single mass range. ${ }^{14,34}$ Each PImMS pixel contains four memory registers, reducing the likelihood of any non-linear response arising from multiple ions being simultaneously detected at the same position. Combining microscope MSI with a fast imaging sensor allows multi-mass ion images to be recorded within minutes, creating a high-throughput alternative to microprobe imaging experiments, which, at identical laser repetition rates, would require the same time to sample one pixel.

The mass spectrometer was modeled using SIMION 8.1 to determine the electrode potentials required to attain its optimal mass and spatial resolutions. ${ }^{35}$ A genetic algorithm was used to determine each parameter. $^{36}$ This approach constrained the potential of the electrodes to their maximum available voltages and randomized their values to generate an initial population of variables. Combinations that resulted in improved mass or spatial resolutions were then given a higher probability of breeding subsequent populations. This process was repeated until no further improvements were observed and revealed that, over the voltage range used, it is possible to individually optimize parameters to increase the mass or spatial resolution of 
the instrument. As expected intuitively, the spatial resolution is primarily controlled by the ion microscope (i.e., the extractor and einzel lens potentials), whereas the mass resolution is affected mainly by the reflectron potential and PEDA pulse.

\section{RESULTS AND DISCUSSION}

Seven commercial dyes spanning 200-700 Da (Auramine O, Coumarin 450, Exalite 404, Rhodamine B, Rhodamine 6G chloride, Rhodamine 560 chloride, and Rhodamine 640 perchlorate) were individually electrosprayed through a nickel mesh onto conductive ITO plates, producing grid patterned samples with approximately $40 \mu \mathrm{m}$ widths and $63 \mu \mathrm{m}$ pitches. Time-of-flight spectra and ion images were then recorded for each sample to determine the mass and spatial resolution, respectively, of the instrument as a function of $\mathrm{m} / z$.

\section{A. Mass resolution}

The ion microscope focuses a particular $\mathrm{m} / \mathrm{z}$ and centers the effective mass range of the instrument around this point. The focused $\mathrm{m} / z$ can be changed by adjusting the potentials applied to each electrode using the ratio $(\mathrm{m} / z)_{2} /(\mathrm{m} / z)_{1}$, where $(\mathrm{m} / z)_{1}$ is the initially optimized ion and $(\mathrm{m} / z)_{2}$ is the ion to be focused. ${ }^{37}$ Higher potentials, therefore, focus ions with larger $\mathrm{m} / z$, and vice versa. Figure 2(a) presents the laser desorption ionization mass spectrum of Rhodamine B, optimized for its most intense peak at $343 \mathrm{Da}$ and averaged over 2000 laser shots. The inset of Fig. 2(a) demonstrates that the mass resolution of this peak, determined from the full width at half maximum (FWHM) of a fitted Gaussian function, is 8100 $\pm 700 \mathrm{~m} / \Delta \mathrm{m}$. To achieve this resolution, the repeller, extractor, and central einzel lens potentials were set to $U_{\mathrm{Re}}=5.000 \mathrm{kV}$, $U_{\mathrm{Ex}}=4.680 \mathrm{kV}$, and $U_{\mathrm{Ei}}=1.715 \mathrm{kV}$, respectively. The applied PEDA potential was $U_{\mathrm{P}}=5.300 \mathrm{kV}$, and the deflector and reflectron voltages were held at $U_{\mathrm{D}}= \pm 55 \mathrm{~V}$ and $U_{\mathrm{R}}=8.750 \mathrm{kV}$. The PEDA pulse was delayed $2.85 \mu$ s relative to the laser pulse. These parameters only differ by approximately $2 \%$ from those predicted by SIMION 8.1 .
Figure 2(b) illustrates how the instrument mass resolution changes with $\mathrm{m} / z$ while optimized for $343 \mathrm{Da}$. simION predicts that the maximum resolution is $30200 \pm 2900 \mathrm{~m} / \Delta \mathrm{m}$ at this point and that it halves within $\pm 25 \mathrm{Da}$ (solid gray line). However, convolving the simulation with the $3.7 \mathrm{~ns}$ response time of the detector (dashed gray line) indicates that the expected measurable resolution can at best only be $\sim 8000 \mathrm{~m} / \Delta \mathrm{m}$, matching the experimentally determined value. This effect may also explain why three of the four $m / z$ peaks observed in Fig. 2(a) have comparable mass resolutions (red circles). By contrast, peaks observed under identical conditions for the remaining six dyes lie outside the effective mass range and demonstrate better agreement with the predicted behavior.

The results presented in Fig. 2 were acquired using reflectron and PEDA step potentials of $U_{\mathrm{R}}=8.750 \mathrm{kV}$ and $U_{\mathrm{P}}=5.300 \mathrm{kV}$. To confirm these were the optimal conditions, the attainable mass resolutions for 64 combinations of the reflectron and PEDA step potentials were simulated and recorded experimentally in $0.250 \mathrm{kV}$ steps over $U_{\mathrm{R}}=8.000-9.750 \mathrm{kV}$ and $0.050 \mathrm{kV}$ steps over $U_{\mathrm{P}}=5.100$ $5.450 \mathrm{kV}$. The results are illustrated in Figs. 3(a) and 3(b). Both data sets peak at $U_{\mathrm{R}}=8.750 \mathrm{kV}$ and only vary in $U_{\mathrm{P}}$ by one step. A comparison of the experimental data in Fig. 3(a) (blue and green circles) with the convoluted simulations (red circles) demonstrates excellent agreement for the change in PEDA voltage. However, the reflectron potential exhibits a flatter profile, illustrating the resolution limit imposed by the detector.

The green and blue traces intersecting the maxima in Figs. 3(a) and 3(b) demonstrate that these points represent the measured and simulated conditions under which the reflectron successfully focuses the PEDA pseudo-source plane onto the detector. The blue trace depicts how the mass resolution depends on the reflectron potential and illustrates the voltage at which the first-order energy-focusing condition is satisfied. ${ }^{23}$ By contrast, the green curve demonstrates the expected result that changing the PEDA step potential moves the location of the PEDA temporal focal plane. As the reflectron is an ion mirror, this plane will have a corresponding focal plane after the reflectron exit, which should be tuned to overlap with the detector.
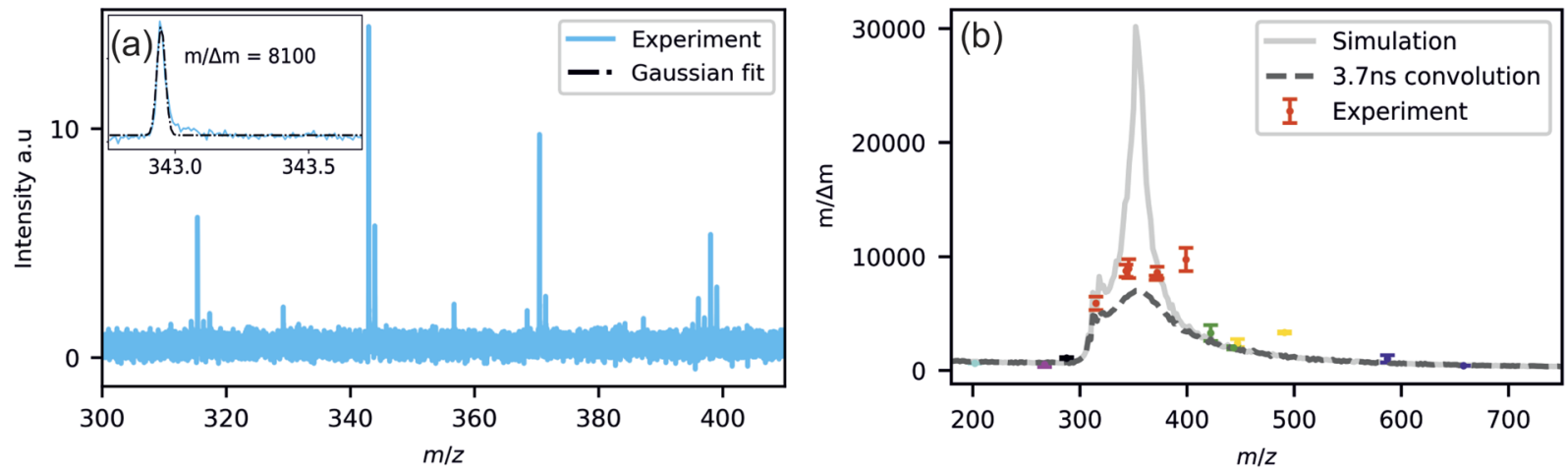

FIG. 2. (a) The Rhodamine B mass spectrum (blue), averaged over 2000 laser shots and optimized for 343 Da using the optimized potentials in Fig. 3 . Fitting this peak with a Gaussian function (black) returns a mass resolution of $8100 \pm 700 \mathrm{~m} / \Delta \mathrm{m}$. (b) The mass resolution dependence on $\mathrm{m} / \mathrm{z}$ when focused for $343 \mathrm{Da}$, categorized using seven dyes spanning a 500 Da range (cyan = Coumarin 450, purple $=$ Rhodamine 560 chloride, black $=$ Auromine 0 , red $=$ Rhodamine $B$, green $=$ Rhodamine $6 \mathrm{G}$ chloride, yellow $=$ Rhodamine 640 perchlorate, and blue = Exalite 404). The measured resolution is limited by the $3.7 \mathrm{~ns}$ response time of the detector (dashed gray line), as demonstrated by convolving this constraint with the mass resolution dependence simulated using SIMION 8.1 (solid gray line). The mass resolved window can be tuned to focus different $m / z$ by applying a linear scaling parameter to each electrode in the ion microscope. 

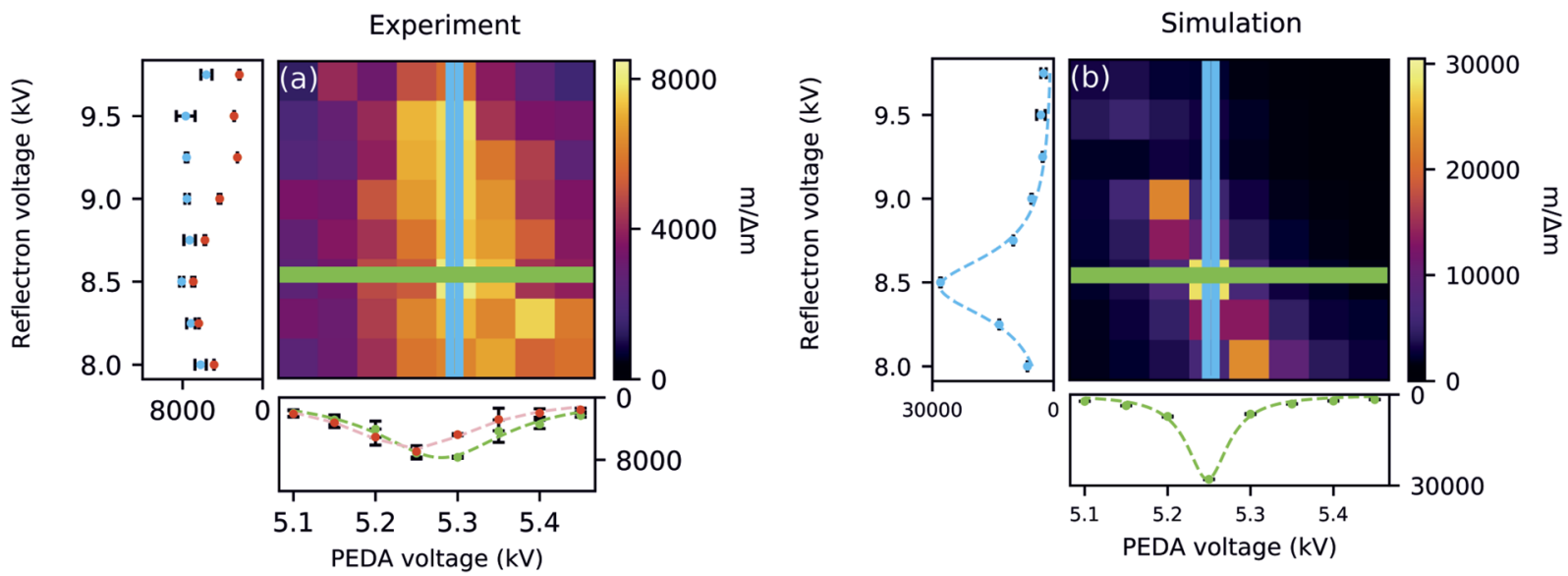

FIG. 3. The measured (a) and simulated (b) mass resolutions as functions of the applied PEDA $\left(U_{P}\right)$ and reflectron potentials $\left(U_{R}\right)$, when focused for 343 Da. The global maxima represent the measured $(8100 \pm 700 \mathrm{~m} / \mathrm{\Delta m})$ and simulated $(30200 \pm 2900 \mathrm{~m} / \mathrm{\Delta m})$ points at which the PEDA pseudo-source plane is focused onto the detector by the reflectron. The blue and green traces crossing these maxima illustrate how the instrument depends on each parameter, as detailed in the text. Projections of each trace (blue and green circles) demonstrate the mass resolution dependence on $U_{R}$ and $U_{p}$. The experimental results are comparable to the simulated data when the latter is convolved with the $3.7 \mathrm{~ns}$ response time of the detector (red circles).

For a fixed reflectron potential, increasing the PEDA voltage results in faster ions and a longer distance between the first temporal focus and reflectron entrance. This moves the corresponding focal plane nearer to the reflectron exit, and vice versa.

Figures 3(a) and 3(b) additionally reveal an inverse relationship between the applied reflectron and PEDA step potentials. This can again be understood in terms of the first-order energy-focusing condition of a single-stage reflectron. As noted above, increasing the applied PEDA potential raises the average kinetic energy $U$ of the focused $\mathrm{m} / z$ packet. This lengthens the field-free drift distance $L_{1}$ between the PEDA temporal focal plane and the detector, as shown in Table I. $L_{1}$ can, therefore, be thought of as $L_{1}(U)$. The PEDA timeof-flight equations can similarly be used to show that a small increase in $U$ leads to a greater proportional increase in $L_{1}(U){ }^{26,27}$ As this distance, the length $L_{2}$ between the detector and reflectron exit, and the reflectron length $d_{\mathrm{R}}$ are fixed during a particular experiment [see Fig. 1(c)], a lower electric field strength $E_{\mathrm{R}}$ is needed to satisfy the reflectron focusing condition,

$$
E_{\mathrm{R}}=\frac{U_{\mathrm{R}}}{d_{\mathrm{R}}}=\frac{4 U}{L_{1}(U)+L_{2}} .
$$

TABLE I. The simulated mass resolution dependence on the applied reflectron and PEDA step potentials.

\begin{tabular}{lccc}
\hline \hline$U_{\mathrm{P}}(\mathrm{kV})^{\mathrm{a}}$ & $U_{\mathrm{R}}(\mathrm{kV})$ & $L_{1}(\mathrm{~mm})$ & $m / \Delta m$ \\
\hline 5.150 & 9.000 & 373 & 7100 \\
5.200 & 8.750 & 541 & 18000 \\
5.250 & 8.500 & 672 & 30200 \\
5.300 & 8.250 & 759 & 17700 \\
5.350 & 8.000 & 825 & 8900 \\
\hline \hline
\end{tabular}

${ }^{\mathrm{a}} U \simeq U_{\mathrm{P}}$.
The above trend appears in both the simulated and experimental data, although the latter is again obscured by the $3.7 \mathrm{~ns}$ response time of the detector.

Table I demonstrates that the attainable mass resolution reaches a maximum value along the trend line described in Fig. 3(b). This at first appears to contradict the first-order energy-focusing condition of the reflectron, which allows the total drift distance $L_{1}$ $+L_{2}$ to be distributed without any conditions so long as Eq. (4) is satisfied. However, this discrepancy can be attributed to the fact that, for practical purposes, the deflector potential, reflectron angle, and detector angle were fixed in the experiments and simulations presented in Fig. 3. Adjusting these parameters can eliminate this effect. One consideration is that increasing the applied PEDA step potential results in faster ions that are deflected at shallower angles by the deflector. These emerge from the reflectron along a different axis, necessitating a change in the reflectron or detector angles to measure the optimal mass resolution. For example, SIMION predicts that modifying the detector position $L_{2}$ and angle $\theta_{\mathrm{D}}$ by $\sim 2 \%$ each allows the maximum simulated mass resolution to be matched within its standard deviation at $U_{\mathrm{R}}=8.250 \mathrm{kV}$ and $U_{\mathrm{P}}=5.300 \mathrm{kV}$, significantly increasing the value of $17700 \mathrm{~m} / \Delta \mathrm{m}$ quoted in Table I.

\section{B. Spatial resolution}

The instrument spatial resolution, subject to the potentials described above, was determined from gridded dye images acquired by gating the $768 \times 568$ pixel CCD camera over the relevant timeof-flight ranges. ${ }^{7,9}$ The integrated ion image of the four Rhodamine B peaks shown in Fig. 2 demonstrates this process. First, the raw data were centroided during the image acquisition [Fig. 4(a)] so that one flash of light on the scintillator corresponded to one pixel event. A Fourier transform was then applied to remove electronic noise introduced by the camera [Fig. 4(b)]. Finally, intensity profiles running perpendicular to the wire silhouettes in the images were 
extracted and fit with rectangular functions to match the grid pattern [Fig. 4(d)]. These functions were each convolved with a Gaussian function and with a linear function that accounts for the $\sim 20^{\circ}$ incidence angle of the laser pulse with respect to the sample surface, and
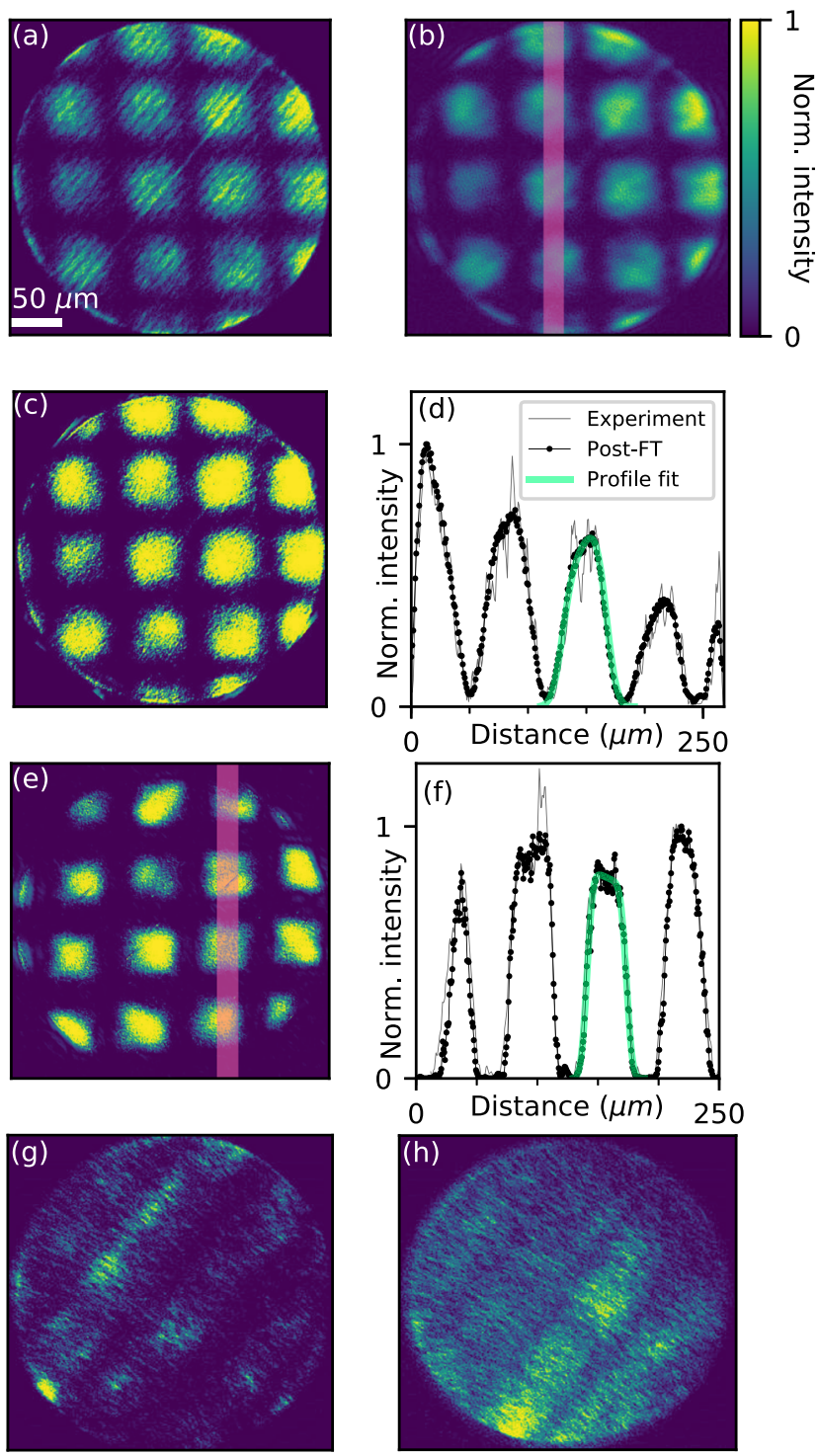

FIG. 4. The centroided CCD image of Rhodamine B (a), gated on the four peaks shown in Fig. 2(a), is processed to determine the instrument spatial resolution. Applying a Fourier transform to the image removes unwanted artifacts (b). Adjusting the image contrast demonstrates the clarity of the resulting grid pattern (c). The spatial resolution is determined from the $20 \%-80 \%$ intensity rises observed along profiles running perpendicular to the wire silhouettes in (b). Each profile is fit using a Gaussian function convolved with a rectangular function representing the grid and a linear function that accounts for the $20^{\circ}$ incidence angle of the laser (d). Increasing the applied extraction field improves the spatial resolution [(e) and (f)]. The stigmatic image focus additionally depends on the reflectron angle relative to the initial time-of-flight axis $\left(\theta_{R}\right)$. This angle is $0.72^{\circ}$ in $(a)-(f)$; slightly adjusting this to $-0.30^{\circ}(\mathrm{g})$ or $2.08^{\circ}(\mathrm{h})$ distorts the image. the latter causes shadowing and hence decreasing intensity across each grid.

The spatial resolution $R_{\mathrm{s}}$ was then determined by averaging the $20 \%-80 \%$ intensity rise of each dye grid and dividing the result by the image magnification $M$ such that

$$
R_{\mathrm{s}}=\frac{1.68 \sigma}{M}
$$

where $\sigma$ is the Gaussian standard deviation. The image magnification was measured by comparing the pitch of the imaged grid pattern with the $63 \mu \mathrm{m}$ pitch of the nickel mesh. In Fig. 4, the ion images were magnified by a factor of $\sim 93$, which corresponds to a $\sim 270 \mu \mathrm{m}$ diameter field of view. The calculated $R_{\mathrm{s}}$ is likely a conservative upper bound, as Eq. (5) represents a limiting case where the grid pattern width is at least six times larger than $\sigma$. In these experiments, the ratio is closer to 3.3, suggesting that the spatial resolution could be up to $10 \%$ better than reported.

The spatial resolution determined from the fitted peak in Fig. 4(d) is $11.95 \mu \mathrm{m} \pm 0.25 \mu \mathrm{m}$. This can be further improved at the expense of the mass resolution by strengthening the applied extraction field. Figures $4(\mathrm{e})$ and $4(\mathrm{f})$ demonstrate that increasing the field from $22.1 \mathrm{~V} \mathrm{~mm}^{-1}$ to $25.9 \mathrm{~V} \mathrm{~mm}^{-1}$ improves the resolution to $6.44 \mu \mathrm{m} \pm 0.14 \mu \mathrm{m}$. In this example, the instrument parameters were set to $U_{\mathrm{Re}}=6.295 \mathrm{kV}, U_{\mathrm{Ex}}=5.920 \mathrm{kV}, U_{\mathrm{Ei}}=2.000 \mathrm{kV}$, and $U_{\mathrm{D}}= \pm 61 \mathrm{~V}$ following an initial simion optimization. The PEDA potential was also increased to $U_{\mathrm{P}}=6.730 \mathrm{kV}$, and its delay relative to the laser pulse was shortened to $2.65 \mu \mathrm{s}$. The reflection potential was increased to $U_{\mathrm{R}}=9.800 \mathrm{kV}$. Better spatial resolution can, in principle, be achieved by continuing to increase the extraction field in this manner. Figure 4 also demonstrates how the reflectron angle $\theta_{\mathrm{R}}$ controls the stigmatic imaging ability of the instrument. Figure $4(\mathrm{~b})$ was acquired at $\theta_{\mathrm{R}}=0.72^{\circ}$. By contrast, Figs. $4(\mathrm{~g})$ and $4(\mathrm{~h})$ were imaged with reflectron angles of $-0.30^{\circ}$ and $2.08^{\circ}$, respectively, demonstrating that when all other parameters are held constant, a small change in this angle distorts the recorded image.

CCD images of each major ToF peak belonging to the seven dyes were additionally used to determine the instrument spatial resolution as a function of $\mathrm{m} / z$. Figure 5 illustrates this dependence over a 200-700 Da range when optimized for 343 Da using the same conditions as in Fig. 2. Similar to the mass resolution, the focused ion can be easily adjusted by applying a scaling parameter $(\mathrm{m} / \mathrm{z})_{2} /(\mathrm{m} / \mathrm{z})_{1}$ to each electrode in the microscope. ${ }^{37}$ Between $300 \mathrm{Da}$ and $600 \mathrm{Da}$, the average spatial resolution is $18 \pm 6 \mu \mathrm{m}$. Comparing this with Fig. 2 demonstrates that this mass window is approximately six times wider than the $50 \mathrm{Da}$ range over which the mass resolution is at least half of its maximum value. This mass-resolved range can, in principle, be extended to contain $\sim 40 \%$ of the spatially resolved window using the double-field PEDA technique or to cover it completely using electrodynamic PEDA. ${ }^{27,33}$ Incorporating these methods is beyond the scope of the current work, but it is worth noting that the latter only requires an adjustment to the circuit providing the PEDA pulse potential; no instrument modifications are needed.

\section{Multi-mass imaging}

CCD cameras are typically limited to imaging a single time-offlight range during an experiment. As a consequence, images can only be acquired for a single $\mathrm{m} / z$ or integrated over a range of $\mathrm{m} / z$ as 


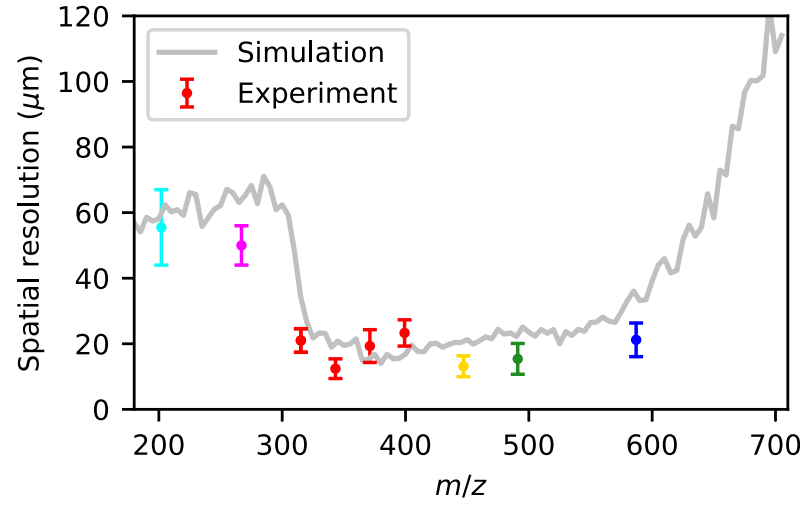

FIG. 5. The spatial resolution dependence on $\mathrm{m} / \mathrm{z}$ when focused for $343 \mathrm{Da}$ using the same conditions as in Fig. 2, categorized using seven dyes (cyan = Coumarin 450 , purple $=$ Rhodamine 560 chloride , black $=$ Auromine 0, red $=$ Rhodamine $\mathrm{B}$, green $=$ Rhodamine $6 \mathrm{G}$ chloride, yellow $=$ Rhodamine 640 perchlorate, and blue $=$ Exalite 404), and compared with simulated data from SIMION 8.1. The spatial resolution is $18 \mu \mathrm{m} \pm 6 \mu \mathrm{m}$ between $300 \mathrm{Da}$ and $600 \mathrm{Da}$. The spatially focused window can easily be shifted to lower or higher $\mathrm{m} / \mathrm{z}$ ranges by applying a linear scaling parameter to each electrode in the ion microscope

is demonstrated in Fig. 4 for Rhodamine B. In that example, a CCD camera was used because its large pixel array allowed the instrument spatial resolution to be more accurately characterized. However, for practical experiments, it is more desirable to perform multi-mass imaging. This can be achieved using event-triggered fast imaging sensors that simultaneously log the positions $(x, y)$ and arrival times $(t)$ of detected events. ${ }^{30-32}$ Integrating the $(x, y, t)$ data over the time-of-flight ranges of interest yields the corresponding images of different $\mathrm{m} / z$.

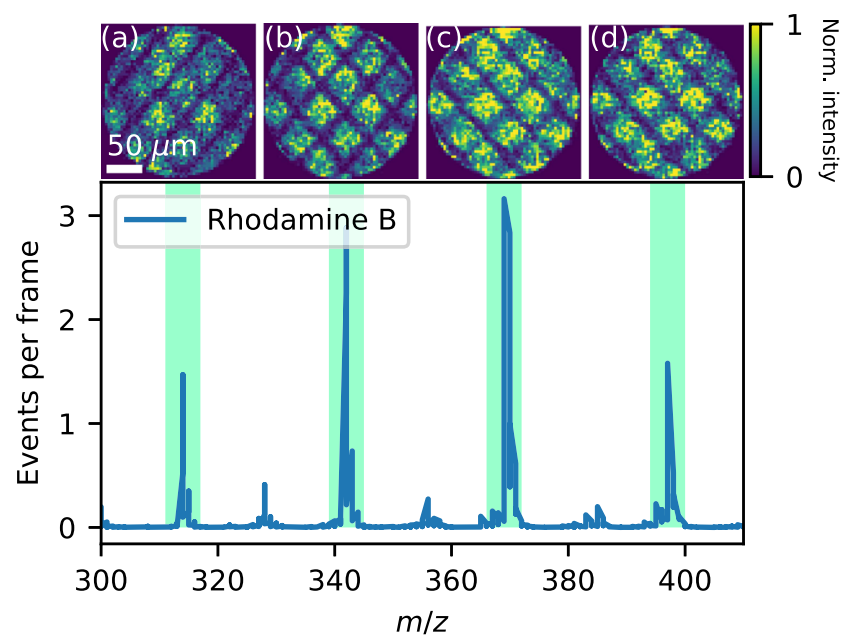

FIG. 6. Multi-mass imaging data acquired using the PImMS1 sensor for Rhodamine $B$ under the same conditions as Fig. 2. Integrating over position $(x, y)$ returns the mass spectrum, while integrating over the shaded time-of-flight peaks creates individual ion images for each $\mathrm{m} / \mathrm{z}[(\mathrm{a})-(\mathrm{d})]$. The $12.5 \mathrm{~ns}$ precision of PImMS1 provides a mass resolution of $1600 \mathrm{~m} / \Delta \mathrm{m}$ at $343 \mathrm{Da}$.
Figure 6 shows the mass spectrum of Rhodamine B recorded using a PImMS fast imaging camera installed with the $72 \times 72$ pixel PImMS1 sensor. The 12.5 ns timing precision of PImMS1 limits the achievable mass resolution in this case to $\sim 1600 \mathrm{~m} / \Delta \mathrm{m}$ but is more than sufficient to resolve ion images for each observed peak. Integrating the $(x, y, t)$ data over the shaded time-of-flight ranges produces the ion images in Figs. 6(a)-6(d). These are consistent with the dependency of spatial resolution on $\mathrm{m} / z$ shown in Fig. 5; Fig. 6(b) is centered on the focused $\mathrm{m} / z$ of $343 \mathrm{Da}$ and is the best resolved.

Developments in fast imaging sensors have increased their achievable timing precision to $1.5 \mathrm{~ns} .^{40}$ This corresponds to a mass resolution of $\sim 24000 \mathrm{~m} / \Delta m$ in vacuum over the mass range of Rhodamine $\mathrm{B}$ and would allow the achievable mass resolution of the reflectron to be fully exploited, paving the way toward ion imaging of complex mass spectra.

\section{CONCLUSION}

A time-of-flight mass spectrometer incorporating an ion microscope and reflectron was used to sequentially image the massresolved ion laser desorbed from a $\sim 270 \mu \mathrm{m}$ diameter surface. The pulsed extraction differential acceleration technique was employed to correct for the initial velocity distribution of the desorbed ions and to create a temporally focused pseudo-source plane for the reflectron. Ion kinetic energies were then additionally focused to the firstorder using the reflectron, resulting in measured mass and spatial resolutions of $8100 \pm 700 \mathrm{~m} / \Delta m$ and $18 \mu \mathrm{m} \pm 6 \mu \mathrm{m}$, respectively. The former is limited only by the $3.7 \mathrm{~ns}$ response time of the microchannel plate/scintillator array used for ion detection. Developments in fast imaging sensors that can be placed in vacuum will remove the need for such detectors, and SIMION suggests that deconvoluting their effect from the measured mass spectra could allow mass resolutions greater than $30000 \mathrm{~m} / \Delta m$ to be achieved. ${ }^{41,42}$ The spatial resolution can also be improved to about $6 \mu \mathrm{m}$ by strengthening the extraction field from $22.1 \mathrm{~V} \mathrm{~mm}^{-1}$ to $25.9 \mathrm{~V} \mathrm{~mm}^{-1}$, and simion predicts that this can be further improved to $500 \mathrm{~nm}$ by increasing the field to approximately $157 \mathrm{~V} \mathrm{~mm}^{-1}$. This would necessarily reduce the achievable mass resolution (to about $2000 \mathrm{~m} / \Delta m$ at 343 $\mathrm{Da}$ ) but would enable more sharply focused images to be rapidly recorded.

The event-triggered PImMS1 sensor was additionally used to demonstrate multi-mass imaging of Rhodamine B peaks over an approximately $100 \mathrm{Da}$ window. In these experiments, the massresolved window over which the instrument reached half of its focused value was approximately $50 \mathrm{Da}$, as compared to the 300 Da range that could be spatially focused to $\sim 20 \mu \mathrm{m}$. This massresolved window is primarily defined by the PEDA technique and could be expanded to cover the entire spatially resolved window using a many-field or electrodynamic PEDA method. ${ }^{27,33}$ Fast imaging sensors such as PImMS, therefore, have the capability to simultaneously image every peak resolved by the reflectron and ion microscope in a single experiment, illustrating the potential of microscope MSI as a high-throughput technique that, when combined with single- or multi-reflecting time-of-flight instruments, could be applied to extract greater information from time-sensitive or irreplaceable samples. 


\section{ACKNOWLEDGMENTS}

The authors gratefully acknowledge the support of the UK EPSRC (Programme Grant No. EP/L005913/1). N.S. thanks the EPSRC and National Physical Laboratory for her iCASE studentship. A.G. is thankful to the China Scholarship Council (CSC) for funding, and R.J.B. is thankful to Jesus College, Oxford, for its financial support.

\section{REFERENCES}

${ }^{1}$ R. Castaing and G. Slodzian, "Microanalyse par émission ionique secondaire," J. Microsc. 1, 395 (1962).

${ }^{2}$ G. Slodzian, "Etude d'une méthode d'analyse locale chimique et isotopique utilisant l'émission ionique secondaire," Ann. Phys. 13, 591-648 (1964).

${ }^{3}$ R. Castaing and G. Slodzian, "Analytical microscopy by secondary ion imaging techniques,” J. Phys. E: Sci. Instrum. 14, 1119-1127 (1981).

${ }^{4}$ B. Schueler, P. Sander, and D. Reed, "A time-of-flight secondary ion microscope," Vacuum 41, 1661-1664 (1990)

${ }^{5}$ S. L. Luxembourg, T. H. Mize, L. A. McDonnell, and R. M. A. Heeren, "Highspatial resolution mass spectrometric imaging of peptide and protein distributions on a surface," Anal. Chem. 76, 5339-5344 (2004).

${ }^{6}$ J. H. Jungmann, A. Gijsbertsen, J. Visser, J. Visschers, R. M. A. Heeren, and M. J. J. Vrakking, "A new imaging method for understanding chemical dynamics: Efficient slice imaging using an in-vacuum pixel detector," Rev. Sci. Instrum. 81, 103112 (2010)

${ }^{7}$ J. H. Jungmann, L. MacAleese, R. Buijs, F. Giskes, A. de Snaijer, J. Visser, J. Visschers, M. J. J. Vrakking, and R. M. A. Heeren, "Fast, high resolution mass spectrometry imaging using a Medipix pixelated detector," J. Am. Soc. Mass Spectrom. 21, 2023-2030 (2010).

${ }^{8}$ M. Brouard, A. J. Johnsen, A. Nomerotski, C. S. Slater, C. Vallance, and W. H. Yuen, "Application of fast sensors to microscope mode spatial imaging mass spectrometry,” J. Instrum. 6, C01044 (2011).

${ }^{9}$ M. Brouard, E. Halford, A. Lauer, C. S. Slater, B. Winter, W. H. Yuen, J. J. John, L. Hill, A. Nomerotski, A. Clark, J. Crooks, I. Sedgwick, R. Turchetta, J. W. L. Lee, C. Vallance, and E. Wilman, "The application of the fast, multi-hit, pixel imaging mass spectrometry sensor to spatial imaging mass spectrometry," Rev. Sci. Instrum. 83, 114101 (2012).

${ }^{10}$ A. Kiss, J. H. Jungmann, D. F. Smith, and R. M. Heeren, "Microscope mode secondary ion mass spectrometry imaging with a Timepix detector," Rev. Sci. Instrum. 84, 013704 (2013).

${ }^{11}$ J. H. Jungmann, D. F. Smith, A. Kiss, L. MacAleese, R. Buijs, and R. M. A. Heeren, "An in-vacuum, pixelated detection system for mass spectrometric analysis and imaging of macromolecules," Int. J. Mass Spectrom. 341, 34-44 (2013).

${ }^{12}$ A. Kiss, D. F. Smith, J. H. Jungmann, and R. M. A. Heeren, "Cluster secondary ion mass spectrometry microscope mode mass spectrometry imaging," Rapid Commun. Mass Spectrom. 27, 2745-2750 (2013).

${ }^{13}$ E. Halford, B. Winter, M. D. Mills, S. P. Thompson, V. Parr, J. J. John, A. Nomerotski, C. Vallance, R. Turchetta, and M. Brouard, "Modifications to a commercially available linear mass spectrometer for mass-resolved microscopy with the pixel imaging mass spectrometry (PImMS) camera," Rapid Commun. Mass Spectrom. 28, 1649-1657 (2014).

${ }^{14}$ C. Vallance, M. Brouard, A. Lauer, C. S. Slater, E. Halford, B. Winter, S. J. King, J. W. L. Lee, D. E. Pooley, I. Sedgwick, R. Turchetta, A. Nomerotski, J. J. John, and L. Hill, "Fast sensors for time-of-flight imaging applications," Phys. Chem. Chem. Phys, 16, 383-395 (2014).

${ }^{15}$ B. W. Schueler, "Microscope imaging by time-of-flight secondary ion mass spectrometry," Microsc., Microanal., Microstruct. 3, 119-139 (1992).

${ }^{16}$ H. Hazama, J. Aoki, H. Nagao, R. Suzuki, T. Tashima, K. Fujii, K. Masuda, K. Awazu, M. Toyoda, and Y. Naito, "Construction of a novel stigmatic MALDI imaging mass spectrometer,” Appl. Surf. Sci. 255, 1257-1263 (2008).

${ }^{17} \mathrm{M}$. Toyoda, "Development of multi-turn time-of-flight mass spectrometers and their applications,” Eur. J. Mass Spectrom. 16, 397-406 (2010).
${ }^{18}$ H. Hazama, H. Yoshimura, K. Awazu, T. Tashima, J. Aoki, M. Toyoda, H. Nagao, K. Masuda, K. Fujii, and Y. Naito, "Development of a stigmatic mass microscope using laser desorption/ionization and a multi-turn time-of-flight mass spectrometer," J. Biomed. Opt. 16, 046007 (2011).

${ }^{19}$ S. G. Alikanov, “A new impulse technique for ion mass measurement," Zh. Eksp. Teor. Fiz. 4, 452 (1957).

${ }^{20}$ B. A. Mamyrin, V. I. Karataev, D. V. Shmikk, and V. A. Zagulin, "The mass-reflectron, a new nonmagnetic time-of-flight mass spectrometer with high resolution," Zh. Eksp. Teor. Fiz. 64, 82-89 (1973).

${ }^{21}$ X. Tang, R. Beavis, W. Ens, F. Lafortune, B. Schueler, and K. G. Standing, “A secondary ion time-of-flight mass spectrometer with an ion mirror," Int. J. Mass Spectrom. Ion Processes 85, 43-67 (1988).

${ }^{22}$ E. V. Moskovets, "Optimization of the reflecting system parameters in the massreflectron,” Appl. Phys. B 53, 253-259 (1991).

${ }^{23}$ B. A. Mamyrin, "Laser assisted reflectron time-of-flight mass spectrometry," Int. J. Mass Spectrom. Ion Processes 131, 1-19 (1994).

${ }^{24}$ M. I. Yavor, T. V. Pomozov, S. N. Kirillov, Y. I. Khasin, and A. N. Verenchikov, "High performance gridless ion mirrors for multi-reflection time-of-flight and electrostatic trap mass analyzers," Int. J. Mass Spectrom. 426, 1-11 (2018).

${ }^{25}$ B. D. Leskiw, M. H. Kim, G. E. Hall, and A. G. Suits, "Reflectron velocity map ion imaging,” Rev. Sci. Instrum. 76, 104101 (2005).

${ }^{26}$ J. Aoki, H. Hazama, and M. Toyoda, "Novel ion extraction method for imaging mass spectrometry,” J. Mass Spectrom. Soc. Jpn. 59, 57-61 (2011).

${ }^{27}$ A. Guo, M. Burt, and M. Brouard, "Mass-resolved ion microscope imaging over expanded mass ranges using double-field post-extraction differential acceleration," Int. J. Mass Spectrom. 429, 121-126 (2018).

${ }^{28}$ T. V. Pomozov, M. I. Yavor, and A. N. Verentchikov, "Reflectrons with ion orthogonal acceleration based on planar gridless mirrors," Tech. Phys. 57, 550555 (2012).

${ }^{29}$ B. Winter, S. King, M. Brouard, and C. Vallance, "A fast microchannel platescintillator detector for velocity map imaging and imaging mass spectrometry," Rev. Sci. Instrum. 85, 023306 (2014).

${ }^{30}$ J. J. John, M. Brouard, A. Clark, J. Crooks, E. Halford, L. Hill, J. W. L. Lee, A. Nomerotski, R. Pisarczyk, I. Sedgwick, C. S. Slater, R. Turchetta, C. Vallance, E. Wilman, B. Winter, and W. H. Yuen, "PImMS, a fast event-triggered monolithic pixel detector with storage of multiple timestamps," J. Instrum. 7, C08001 (2012).

${ }^{31}$ X. Llopart, R. Ballabriga, M. Campbell, L. Tlustos, and W. Wong, "Timepix, a $65 \mathrm{k}$ programmable pixel readout chip for arrival time, energy and/or photon counting measurements," Nucl. Instrum. Methods Phys. Res., Sect. A 581, 485 494 (2007)

${ }^{32}$ T. Poikela, J. Plosila, T. Westerlund, M. Campbell, M. de Gaspari, X. Llopart, V. Gromov, R. Kluit, M. van Beuzekom, F. Zappon, V. Zivkovic, C. Brezina, K. Desch, Y. Fu, and A. Kruth, "Timepix3: A 65k channel hybrid pixel readout chip with simultaneous ToA/ToT and sparse readout," J. Instrum. 9, C05013 (2014).

${ }^{33}$ A. Guo, "Improving the performance of microscope mass spectrometry imaging," Ph.D. thesis, University of Oxford, 2018.

${ }^{34}$ A. T. Clark, J. P. Crooks, I. Sedgwick, R. Turchetta, J. W. L. Lee, J. J. John, E. S. Wilman, L. Hill, E. Halford, C. S. Slater, B. Winter, W. H. Yuen, S. H. Gardiner, M. L. Lipciuc, M. Brouard, A. Nomerotski, and C. Vallance, "Multimass velocitymap imaging with the pixel imaging mass spectrometry (PImMS) sensor: An ultra-fast event-triggered camera for particle imaging," J. Phys. Chem. A 116, 10897-10903 (2012).

${ }^{35}$ D. J. Manura and D. A. Dahl, SIMION Version 8.0/8.1 User Manual, Scientific Instrument Services, Inc., 2011.

${ }^{36}$ Q. J. Wang, "The genetic algorithm and its application to calibrating conceptual rainfall-runoff models," Water Resour. Res. 27, 2467-2471, https://doi.org/ 10.1029/91wr01305 (1991).

${ }^{37}$ B. Winter, E. Halford, and M. Brouard, "Velocity corrected ion extraction in microscope mode imaging mass spectrometry," Int. J. Mass Spectrom. 356, 14-23 (2013).

${ }^{38}$ T. L. Colliver, C. L. Brummel, M. L. Pacholski, F. D. Swanek, A. G. Ewing, and N. Winograd, "Atomic and molecular imaging at the single-cell level with tofsims,” Anal. Chem. 69, 2225-2231 (1997). 
${ }^{39}$ E. Halford, "Microscope-mode imaging mass spectrometry with the PImMS camera," Ph.D. thesis, University of Oxford, 2016

${ }^{40}$ A. Zhao, M. van Beuzekom, B. Bouwens, D. Byelov, I. Chakaberia, C. Cheng, E. Maddox, A. Nomerotski, P. Svihra, J. Visser, V. Vrba, and T. Weinacht, "Coincidence velocity map imaging using Tpx3Cam, a time stamping optical camera with 1.5 ns timing resolution," Rev. Sci. Instrum. 88, 113104 (2017).
${ }^{41}$ E. S. Wilman, S. H. Gardiner, A. Nomerotski, R. Turchetta, M. Brouard, and C. Vallance, "A new detector for mass spectrometry: Direct detection of low energy ions using a multi-pixel photon counter," Rev. Sci. Instrum. 83, 013304 (2012).

${ }^{42}$ B. Winter, S. J. King, M. Brouard, and C. Vallance, "Improved direct detection of low-energy ions using a multipixel photon counter coupled with a novel scintillator," Int. J. Mass Spectrom. 397, 27-31 (2016). 